\title{
Inexact Multistage Stochastic Chance Constrained Programming Model for Water Resources Management under Uncertainties
}

\author{
Hong Zhang, ${ }^{1}$ Minghu Ha, ${ }^{1}$ Hongyu Zhao, ${ }^{2}$ and Jianwei Song ${ }^{3}$ \\ ${ }^{1}$ College of Water Conservancy and Hydropower, School of Science, Hebei University of Engineering, Handan 056038, China \\ ${ }^{2}$ College of Arts, Hebei University of Engineering, Handan 056038, China \\ ${ }^{3}$ School of Economics and Management, Handan University, Handan 056038, China \\ Correspondence should be addressed to Minghu Ha; haminghu@hebeu.edu.cn
}

Received 25 December 2016; Accepted 23 May 2017; Published 20 June 2017

Academic Editor: Fabrizio Riguzzi

Copyright (C) 2017 Hong Zhang et al. This is an open access article distributed under the Creative Commons Attribution License, which permits unrestricted use, distribution, and reproduction in any medium, provided the original work is properly cited.

\begin{abstract}
In order to formulate water allocation schemes under uncertainties in the water resources management systems, an inexact multistage stochastic chance constrained programming (IMSCCP) model is proposed. The model integrates stochastic chance constrained programming, multistage stochastic programming, and inexact stochastic programming within a general optimization framework to handle the uncertainties occurring in both constraints and objective. These uncertainties are expressed as probability distributions, interval with multiply distributed stochastic boundaries, dynamic features of the long-term water allocation plans, and so on. Compared with the existing inexact multistage stochastic programming, the IMSCCP can be used to assess more system risks and handle more complicated uncertainties in water resources management systems. The IMSCCP model is applied to a hypothetical case study of water resources management. In order to construct an approximate solution for the model, a hybrid algorithm, which incorporates stochastic simulation, back propagation neural network, and genetic algorithm, is proposed. The results show that the optimal value represents the maximal net system benefit achieved with a given confidence level under chance constraints, and the solutions provide optimal water allocation schemes to multiple users over a multiperiod planning horizon.
\end{abstract}

\section{Introduction}

With the economic development and population growth, the demands for water resources increase every year. Due to unreasonable exploitation and utilization of water resources, water pollution, and extreme weather conditions, more and more countries and regions are faced with different degrees of water shortage. Making the best use of limited water resources premises the sustainable development of economy and society [1]. In the past few decades, many researchers have applied optimization techniques [2-7] to deal with uncertainties in a number of system components and their interrelationships within water resources systems [8]. Among them, inexact multistage stochastic programming (IMSP) is regarded as a significant method for water resources management. For example, Li et al. [9] proposed an intervalparameter multistage stochastic linear programming model which incorporates inexact optimization and multistage stochastic linear programming to manage water resources.
Zhou et al. [10] developed a factorial multistage stochastic programming which is a hybrid methodology of factorial analysis and IMSP to analyze the potential interrelationships among a variety of uncertain parameters and their impacts on system performance for water resources management. Suo et al. [11] proposed an inventory-theory-based inexact multistage stochastic programming for water resources management through introducing an inventory theory into the framework of IMSP to provide reasonable transferring schemes associated with various flow scenarios. IMSP was also combined with other types of uncertainties, such as fuzziness [12-14], to solve the water resources management problems. However, the above models cannot assess the risks in water resources management systems, to which chance constrained programming (CCP) is an effective method to resort to.

CCP, pioneered by Charnes and Cooper [15], provides a method to handle uncertainties by specifying a confidence level at which it is desired that the constraint holds [16], 
which has been applied in several areas $[17,18]$. In water resources management, using CCP is conducive to not only making decisions of water allocation but also gaining insights into the tradeoffs between economic objectives and some policy factors for a water resource manager [19]. For instance, Liu et al. [1] provided a factorial multistage stochastic programming with chance constraints to deal with the issues of constraint-violation risks and interactive uncertainties in water resources management systems. However, this kind of CCP models can only be solved in some special cases. Meanwhile, the models should firstly be converted into deterministic equivalents and then be solved by using solution methods of deterministic mathematical programming [16] in the solution process. Complex chance constrained programming models are hard to solve, such as nonlinear models which contain multiply distributed data [20]. In order to overcome the limitations, Liu [21] presented a framework of CCP with the assumption that not only uncertain constraints but also uncertain objective would hold at different confidence levels provided by the decision-maker as an appropriate safety margin and gave a hybrid algorithm to solve the CCP for more cases. It is noticeable that the choice of confidence levels makes the model flexible to more situations. The CCP has been applied to many aspects of system decision-making, such as project scheduling [22], capital budgeting [23], capacitated location-allocation [24], and redundancy-optimization [25]. Up to now, Liu [21]'s CCP has not been applied in the optimization of water resources management although this method is needed because of the complexity of the water resources management systems.

In our previous relevant research, [26] extended Liu [21]'s CCP from probability space to Sugeno measure space, in which Sugeno measure was one of representative nonadditive measures. Although the theoretical basis of the CCP was discussed in detail, we did not apply the model to water resources management. Paper [27], which managed water supply risk by constructing a model of water option pricing, did not refer to water resources allocation and assess the risks by using CCP. As an extension of the previous efforts, this study aims to develop an inexact multistage stochastic chance constrained programming (IMSCCP) model, which incorporates the CCP proposed by Liu [21] into the IMSP framework for water resources management. The model can tackle uncertainties in the objective and constraints which present themselves as probability distributions and interval with multiply distributed stochastic boundaries, analyze various policy scenarios when the promised policy targets are violated, and deal with the issue of risks presented as stochastic constraints and stochastic objective with predetermined confidence levels. A hypothetical case study of water resources management within three planning periods is given to demonstrate the applicability of the method. Moreover, a hybrid algorithm incorporating stochastic simulation, back propagation (BP) neural network, and genetic algorithm (GA) is proposed to solve the model. The developed method obtains the results of the model in which the optimal value represents the maximal net system benefit achieved with a given confidence level subject to chance constrains.

\section{Methodologies}

2.1. Multistage Inexact Stochastic Programming Model. It is important for a water resources manager to allocate water to multiple users such as municipal, industrial, and agricultural sectors from an unregulated reservoir over a multiperiod planning horizon in an optimized way, which could be formulated as maximizing the expected economic revenue based on the water allocation in the region over the planning horizon [28]. The manager promises a quantity of water to each user. If the promised water is delivered, net benefits will be brought to local economy; otherwise, the deficient water must be obtained from alternative and more expensive sources or the demand must be curtailed, resulting in economic penalties on local economy [29]. Considering the randomness of the water flow and the dynamic feature of the long-term water allocation plans, the problem of water allocation can be formulated as the following scenario-based multistage stochastic programming model for water resources management under uncertainties [9]:

$$
\begin{array}{ll}
\max & f=\sum_{i=1}^{m} \sum_{j=1}^{n} \mathrm{NB}_{i j} T_{i j}-\sum_{j=1}^{n} E\left[\sum_{i=1}^{m} C_{i j} D_{i Q_{j}}\right] \\
\text { s.t. } & \sum_{i=1}^{m}\left(T_{i j}-D_{i Q_{j}}\right) \leq Q_{j}+\varepsilon_{(j-1) Q_{j-1}}, \\
& \quad j=1,2, \ldots, n \\
& \varepsilon_{(j-1) Q_{j-1}}\left[Q_{j-1}-\sum_{i=1}^{m}\left(T_{i(j-1)}-D_{i Q_{j-1}}\right)\right]+\varepsilon_{(j-2) Q_{j-2}}, \\
& \quad j=1,2, \ldots, n \\
T_{i j \max } \geq T_{i j} \geq D_{i Q_{j}} \geq 0, &
\end{array}
$$

where $f$ is the net benefit of the water allocation system $(\$) ; \mathrm{NB}_{i j}$ is the net benefit when per unit $\left(\mathrm{m}^{3}\right)$ of water is allocated to user $i$ in period $j\left(\$ / \mathrm{m}^{3}\right) ; T_{i j}$ is the fixed amount of water allocation target promised to user $i$ in period $j\left(\mathrm{~m}^{3}\right) ; Q_{j}$ is the water flow in period $j$ which is a random variable $\left(\mathrm{m}^{3}\right) ; D_{i Q_{j}}$ is the amount of water shortage to user $i$ when the seasonal flow is $Q_{j}\left(\mathrm{~m}^{3}\right) ; C_{i j}$ is the loss when per unit $\left(\mathrm{m}^{3}\right)$ of water is not allocated to user $i$ in period $j\left(\$ / \mathrm{m}^{3}\right) ; E[\cdot]$ is the expected value of a random variable; $\varepsilon_{(j-1) Q_{j-1}}$ is the surplus water inflow in period $j\left(\mathrm{~m}^{3}\right)$ according to $Q_{j-1} ; T_{i j \max }$ is the amount of maximum allowable allocation for user $i\left(\mathrm{~m}^{3}\right) ; m$ is the number of water users; $i$ is the index of water user for $i=1,2,3$, with $i=1$ for the municipality, $i=2$ for the industrial user, and $i=3$ for the agricultural sector. In the model, $D_{i Q_{j}}$ is the decision variable and $f$ is the objective function. It is observed that model (1) reflects 
nonanticipativity, since a decision must be made in each stage without the knowledge of realizations of random variables in future stages.

Let $Q_{j}$ take values of $q_{j k}$ with probability $p_{j k}$ for scenarios in each planning period $(j)$, where $p_{j k}$ is the probability of occurrence for scenario $k$ in period $j$ and $\sum_{k=1}^{K_{j}} p_{j k}=1$. Then, we have $E\left[\sum_{i=1}^{m} C_{i} D_{i Q_{i}}\right]=\sum_{i=1}^{m} \sum_{k=1}^{K_{j}} p_{j k} C_{i j} D_{i j k}, j=$ $1,2, \ldots, n$, where $K_{j}$ is the sum of scenarios in period $j ; D_{i j k}$ is the amount of water allocation shortage to user $i$ when the scenario $k$ occurs in period $j$. Then model (1) can be reformulated as follows [9]:

$$
\begin{aligned}
& \max \quad f=\sum_{i=1}^{m} \sum_{j=1}^{n} \mathrm{NB}_{i j} T_{i j}-\sum_{i=1}^{m} \sum_{j=1}^{n} \sum_{k=1}^{K_{j}} p_{j k} C_{i j} D_{i j k} \\
& \text { s.t } \sum_{i=1}^{m}\left(T_{i j}-D_{i j k}\right) \leq Q_{j k}+\varepsilon_{(j-1) k}, \\
& j=1,2, \ldots, n, k=1,2, \ldots, K_{j} \\
& \varepsilon_{(j-1) k} \\
& =\left[Q_{(j-1) k}-\sum_{i=1}^{m}\left(T_{i(j-1)}-D_{i(j-1) k}\right)\right]+\varepsilon_{(j-2) k}, \\
& \quad j=1,2, \ldots, n, k=1,2, \ldots, K_{j} \\
& T_{i j \max } \geq T_{i j} \geq D_{i j k} \geq 0, \\
& i=1,2, \ldots, m, j=1,2, \ldots, n, k=1,2, \ldots, K_{j} .
\end{aligned}
$$

Considering that the uncertainties exist in variables and coefficients, the fixed values of the parameters, such as $T_{i j}$, $\mathrm{NB}_{i j}, C_{i j}$, and $Q_{j k}$, cannot be determined exactly. Thus, the inexact multistage stochastic programming (IMSP) model, which introduces interval parameters into model (2), is proposed as follows [9]:

$$
\begin{gathered}
\max \quad f^{ \pm}=\sum_{i=1}^{m} \sum_{j=1}^{n} \mathrm{NB}_{i j}^{ \pm} T_{i j}^{ \pm}-\sum_{i=1}^{m} \sum_{j=1}^{n} \sum_{k=1}^{K_{j}} p_{j k} C_{i j}^{ \pm} D_{i j k}^{ \pm} \\
\text {s.t } \quad \sum_{i=1}^{m}\left(T_{i j}^{ \pm}-D_{i j k}^{ \pm}\right) \leq q_{j k}^{ \pm}+\varepsilon_{(j-1) k}^{ \pm}, \\
\quad j=1,2, \ldots, n, k=1,2, \ldots, K_{j} \\
\varepsilon_{(j-1) k}^{ \pm} \\
=\left[q_{(j-1) k}^{ \pm}-\sum_{i=1}^{m}\left(T_{i(j-1)}^{ \pm}-D_{i(j-1) k}^{ \pm}\right)\right]+\varepsilon_{(j-2) k}^{ \pm}, \\
\quad j=2,3, \ldots, n, k=1,2, \ldots, K_{j} \\
T_{i j \max }^{ \pm} \geq T_{i j}^{ \pm} \geq D_{i j k}^{ \pm} \geq 0, \\
i=1,2, \ldots, m, j=1,2, \ldots, n, k=1,2, \ldots, K_{j} .
\end{gathered}
$$

2.2. Inexact Multistage Stochastic Chance Constrained Programming Model. Models (1), (2), and (3) do not readily assess the risks, and they only deal with uncertainties in the right hand side such as the water flow $Q$. It is difficult to handle uncertainties in both the left and right hand sides (i.e., $T_{i j}, \mathrm{NB}_{i j}$, and $C_{i j}$ ) [1] which are presented as interval with stochastic normal distributed boundaries. In view of the above considerations, Liu et al. [1] combined chance constrained programming (CCP, initiated by Charnes and Cooper [15]) with IMSP to propose the following inexact multistage stochastic programming model with chance constraints to solve problems with the request that chance constraints should hold at least with prescribed levels of probability (i.e., confidence levels):

$$
\begin{gathered}
\max \quad f^{ \pm}=\sum_{i=1}^{m} \sum_{j=1}^{n} \mathrm{NB}_{i j}^{ \pm} T_{i j}^{ \pm}-\sum_{i=1}^{m} \sum_{j=1}^{n} \sum_{k=1}^{K_{j}} p_{j k} C_{i j}^{ \pm} D_{i j k}^{ \pm} \\
\text {s.t } \quad \operatorname{Pr}\left\{\sum_{i=1}^{m}\left(T_{i j}^{ \pm}-D_{i j k}^{ \pm}\right) \leq q_{j k}^{ \pm}+\varepsilon_{(j-1) k}^{ \pm}\right\} \geq \beta_{j k}, \\
j=1,2, \ldots, n, k=1,2, \ldots, K_{j} \\
\varepsilon_{(j-1) k}^{ \pm} \\
=\left[q_{(j-1) k}^{ \pm}-\sum_{i=1}^{m}\left(T_{i(j-1)}^{ \pm}-D_{i(j-1) k}^{ \pm}\right)\right]+\varepsilon_{(j-2) k}^{ \pm}, \\
j=2,3, \ldots, n, k=1,2, \ldots, K_{j} \\
T_{i j \max }^{ \pm} \geq T_{i j}^{ \pm} \geq D_{i j k}^{ \pm} \geq 0, \\
i=1,2, \ldots, m, j=1,2, \ldots, n, k=1,2, \ldots, K_{j} .
\end{gathered}
$$

Model (4) can handle uncertainties presented as interval with normally distributed boundaries. However, uncertainties in the water resources management systems have more diverse forms of performance besides normal distribution, which suggests a need for models that can handle uncertainties presented as interval with multiple distributed boundaries. And the confidence levels only in constraints may be not enough to indicate the relationship between the economic objective and the system risk. Moreover, a water resources manager may want to obtain the maximum that the objective function $f(\mathbf{x}, \boldsymbol{\xi})$ achieves with a given confidence level subject to stochastic constraints with other confidence levels, which means that the confidence levels are not only in constraints but also in objective.

Liu [21] proposed a framework of nonlinear chance constrained programming with confidence levels occurring in constraints and objective and provided a stochastic simulation based genetic algorithm to solve the CCP. This CCP model can be formulated as follows: 


$$
\begin{aligned}
\max & \bar{f} \\
\text { s.t } & \operatorname{Pr}\{\xi \mid f(\mathbf{x}, \boldsymbol{\xi}) \geq \bar{f}\} \geq \alpha \\
& \operatorname{Pr}\left\{\xi \mid g_{j}(\mathbf{x}, \xi) \leq 0\right\} \geq \beta_{j}, \quad j=1,2, \ldots, n,
\end{aligned}
$$

where $\mathbf{x}$ is a decision vector; $\boldsymbol{\xi}$ is a random vector; $\alpha$ and $\beta_{j}$ are predetermined confidence levels for stochastic objective and stochastic constraint(s), respectively; $\operatorname{Pr}\{\cdot\}$ denotes the probability of the event in $\{\cdot\}$. This programming aims to maximize $\bar{f}$ that the objective function $f(\mathbf{x}, \boldsymbol{\xi})$ achieves with at least probability of $\alpha(\max \bar{f}$ is called $\alpha$-optimistic value to $f(\mathbf{x}, \boldsymbol{\xi}))$. $\mathbf{x}$ is feasible if and only if the probability measure of the set $\left\{\xi \mid g_{j}(\mathbf{x}, \xi) \leq 0\right\}$ is at least $\beta_{j}$ for $j=1,2, \ldots, n$.

By incorporating CCP [21] and multistage stochastic programming (model (2)), a multistage stochastic chance constrained programming model for water resources management could be formulated as follows:

$$
\begin{array}{ll}
\max & \bar{f} \\
\text { s.t } & \operatorname{Pr}\left\{\sum_{i=1}^{m} \sum_{j=1}^{n} \mathrm{NB}_{i j} T_{i j}-\sum_{i=1}^{m} \sum_{j=1}^{n} \sum_{k=1}^{K_{j}} p_{j k} C_{i j} D_{i j k} \geq \bar{f}\right\} \\
& \geq \alpha \\
& \operatorname{Pr}\left\{\sum_{i=1}^{m}\left(T_{i j}-D_{i j k}\right) \leq q_{j k}+\varepsilon_{(j-1) k}\right\} \geq \beta_{j k}, \\
& \varepsilon_{(j-1) k}=\left[q_{(j-1) k}-\sum_{i=1}^{m}\left(T_{i(j-1)}-D_{i(j-1)}\right)\right]+\varepsilon_{(j-2) k}, \\
\quad j=2,3, \ldots, n, k=1,2, \ldots, K_{j} & T_{i j \max } \geq T_{i j} \geq D_{i j k} \geq 0, \\
i=1,2, \ldots, m, \quad j=1,2, \ldots, n, k=1,2, \ldots, K_{j},
\end{array}
$$

$\max \bar{f}^{ \pm}$

$$
\begin{array}{ll}
\text { s.t } & \operatorname{Pr}\left\{\sum_{i=1}^{m} \sum_{j=1}^{n} \mathrm{NB}_{i j}^{ \pm}\left(T_{i j}^{-}+\Delta T_{i j} y_{i j}\right)-\sum_{i=1}^{m} \sum_{j=1}^{n} \sum_{k=1}^{K_{j}} p_{j k} C_{i j}^{ \pm} D_{i j k}^{ \pm} \geq \bar{f}^{ \pm}\right\} \geq \alpha \\
& \operatorname{Pr}\left\{\sum_{i=1}^{m}\left(T_{i j}^{-}+\Delta T_{i j} y_{i j}-D_{i j k}^{ \pm}\right) \leq q_{j k}^{ \pm}+\varepsilon_{(j-1) k}^{ \pm}\right\} \geq \beta_{j k}, \quad j=1,2, \ldots, n, k=1,2, \ldots, K_{j} \\
& \mathcal{E}_{(j-1) k}^{ \pm}=\left[q_{(j-1) k}^{ \pm}-\sum_{i=1}^{m}\left(T_{i(j-1)}^{-}+\Delta T_{i(j-1)} y_{i(j-1)}-D_{i(j-1)}^{ \pm}\right)\right]+\varepsilon_{(j-2) k}^{ \pm}, j=2,3, \ldots, n, k=1,2, \ldots, K_{j} \\
& 0 \leq y_{i j} \leq 1, \quad i=1,2, \ldots, m, j=1,2, \ldots, n \\
& T_{i j \max }^{ \pm} \geq T_{i j}^{-}+\Delta T_{i j} y_{i j} \geq D_{i j k}^{ \pm} \geq 0, \quad i=1,2, \ldots, m, j=1,2, \ldots, n, k=1,2, \ldots, K_{j} .
\end{array}
$$

where $\alpha$ and $\beta_{j k}$ are predetermined confidence levels for stochastic objective and stochastic constraint(s), respectively. This programming aims to obtain the $\alpha$-optimistic value to the objective function of $\sum_{i=1}^{m} \sum_{j=1}^{n} \mathrm{NB}_{i j} T_{i j}-$ $\sum_{i=1}^{m} \sum_{j=1}^{n} \sum_{k=1}^{K_{j}} p_{j k} C_{i j} D_{i j k}$. Obviously, model (6) gives predetermined confidence levels for constraints and objective, which indicates more comprehensive risk assessment in the water resources management systems.

Considering that the fixed values of the parameters in model (6) cannot be determined exactly, an inexact multistage stochastic chance constrained programming (IMSCCP) model is proposed as follows:

$$
\begin{array}{ll}
\max & \bar{f}^{ \pm} \\
\text {s.t } & \operatorname{Pr}\left\{\sum_{i=1}^{m} \sum_{j=1}^{n} \mathrm{NB} B_{i j}^{ \pm} T_{i j}^{ \pm}-\sum_{i=1}^{m} \sum_{j=1}^{n} \sum_{k=1}^{K_{j}} p_{j k} C_{i j}^{ \pm} D_{i j k}^{ \pm} \geq \bar{f}^{ \pm}\right\} \\
& \geq \alpha \\
& \operatorname{Pr}\left\{\sum_{i=1}^{m}\left(T_{i j}^{ \pm}-D_{i j k}^{ \pm}\right) \leq q_{j k}^{ \pm}+\varepsilon_{(j-1) k}^{ \pm}\right\} \geq \beta_{j k}, \\
& \varepsilon_{(j-1) k}^{ \pm}=\left[q_{(j-1) k}^{ \pm}-\sum_{i=1}^{m}\left(T_{i(j-1)}^{ \pm}-D_{i(j-1)}^{ \pm}\right)\right]+\varepsilon_{(j-2) k}^{ \pm}, \\
& T_{i j \max }^{ \pm} \geq T_{i j}^{ \pm} \geq D_{i j k}^{ \pm} \geq 0, \ldots, n, k=1,2, \ldots, K_{j} \\
i=1,2, \ldots, m, \quad j=1,2, \ldots, n, k=1,2, \ldots, K_{j} .
\end{array}
$$
$1,2, \ldots, m, j=1,2, \ldots, n$. Model (7) could be replaced by the following form: 
Next, we can change model (8) into two submodels corresponding to the upper and lower bounds for the desired objective function value.
Firstly, we formulate submodel (9) corresponding to the upper bound of the objective function value; that is,

$$
\begin{array}{ll}
\max & \bar{f}^{+} \\
\text {s.t } & \operatorname{Pr}\left\{\sum_{i=1}^{m} \sum_{j=1}^{n} \mathrm{NB}_{i j}^{+}\left(T_{i j}^{-}+\Delta T_{i j} y_{i j}\right)-\sum_{i=1}^{m} \sum_{j=1}^{n} \sum_{k=1}^{K_{j}} p_{j k} C_{i j}^{-} D_{i j k}^{-} \geq \bar{f}^{+}\right\} \geq \alpha \\
& \operatorname{Pr}\left\{\sum_{i=1}^{m}\left(T_{i j}^{-}+\Delta T_{i j} y_{i j}-D_{i j k}^{-}\right) \leq q_{j k}^{+}+\varepsilon_{(j-1) k}^{+}\right\} \geq \beta_{j k}, \quad j=1,2, \ldots, n, k=1,2, \ldots, K_{j} \\
& \varepsilon_{(j-1) k}^{+}=\left[q_{(j-1) k}^{+}-\sum_{i=1}^{m}\left(T_{i(j-1)}^{-}+\Delta T_{i(j-1)} y_{i(j-1)}-D_{i(j-1)}^{-}\right)\right]+\varepsilon_{(j-2) k}^{+}, \quad j=2,3, \ldots, n, k=1,2, \ldots, K_{j} \\
& 0 \leq y_{i j} \leq 1, \quad i=1,2, \ldots, m, j=1,2, \ldots, n \\
& T_{i j \max }^{+} \geq T_{i j}^{-}+\Delta T_{i j} y_{i j} \geq D_{i j k}^{-} \geq 0, \quad i=1,2, \ldots, m, j=1,2, \ldots, n, k=1,2, \ldots, K_{j},
\end{array}
$$

where $D_{i j k}^{-}$and $y_{i j}$ are the decision variables and $\max \bar{f}^{+}$ is the $\alpha$-optimistic value to the net system benefit $\sum_{i=1}^{m} \sum_{j=1}^{n} \mathrm{NB}_{i j}^{+}\left(T_{i j}^{-}+\Delta T_{i j} y_{i j}\right)-\sum_{i=1}^{m} \sum_{j=1}^{n} \sum_{k=1}^{K_{j}} p_{j k} C_{i j}^{-} D_{i j k}^{-}$. In submodel (9), the optimal solutions are denoted by $D_{i j k o p t}^{-}$ and $y_{i j o p t}$, and the optimal value is denoted by $\bar{f}_{\text {opt }}^{+}$.
Secondly, submodel (10) corresponding to the lower bound of the objective function value can be formulated as follows:

$$
\begin{array}{ll}
\max & \bar{f} \\
\text { s.t } & \operatorname{Pr}\left\{\sum_{i=1}^{m} \sum_{j=1}^{n} \mathrm{NB}_{i j}^{-}\left(T_{i j}^{-}+\Delta T_{i j} y_{i j \mathrm{opt}}\right)-\sum_{i=1}^{m} \sum_{j=1}^{n} \sum_{k=1}^{K_{j}} p_{j k} C_{i j}^{+} D_{i j k}^{+} \geq \bar{f}_{\mathrm{opt}}^{+}\right\} \geq \alpha \\
& \operatorname{Pr}\left\{\sum_{i=1}^{m}\left(T_{i j}^{-}+\Delta T_{i j} y_{i j \mathrm{opt}}-D_{i j k}^{+}\right) \leq q_{j k}^{-}+\varepsilon_{(j-1) k}^{-}\right\} \geq \beta_{j k}, \quad j=1,2, \ldots, n, k=1,2, \ldots, K_{j} \\
& \mathcal{E}_{(j-1) k}^{-}=\left[q_{(j-1) k}^{-}-\sum_{i=1}^{m}\left(T_{i(j-1)}^{-}+\Delta T_{i(j-1)} y_{i(j-1) \mathrm{opt}}-D_{i(j-1)}^{+}\right)\right]+\varepsilon_{(j-2) k}^{-}, \quad j=2,3, \ldots, n, k=1,2, \ldots, K_{j} \\
& T_{i j}^{-}+\Delta T_{i j} y_{i j \mathrm{opt}} \geq D_{i j k}^{+} \geq D_{i j k \mathrm{opt}}^{-}, \quad i=1,2, \ldots, m, j=1,2, \ldots, n, k=1,2, \ldots, K_{j},
\end{array}
$$

where $D_{i j k}^{+}$is the decision variable and $\max \bar{f}^{-}$is the $\alpha$ optimistic value to the net system benefit $\sum_{i=1}^{m} \sum_{j=1}^{n} \mathrm{NB}_{i j}^{-}\left(T_{i j}^{-}+\right.$ $\left.\Delta T_{i j} y_{i j o p t}\right)-\sum_{i=1}^{m} \sum_{j=1}^{n} \sum_{k=1}^{K_{j}} p_{j k} C_{i j}^{+} D_{i j k}^{+}$. In submodel (10), the optimal solution is denoted by $D_{i j k \text { opt }}^{+}$and the optimal value is denote by $\bar{f}_{\text {opt }}^{-}$.

Thirdly, the real allocation of water to user $i$ when the scenario $k$ occurs in period $j$ can be calculated by $A_{i j k \text { opt }}^{ \pm}=$ $T_{i j}^{-}+\Delta T_{i j} y_{i j \mathrm{opt}}-D_{i j k \mathrm{opt}}^{ \pm}$.
Finally, the optimized interval solution for the decision variable $D_{i j k \mathrm{opt}}^{ \pm}=\left[D_{i j \mathrm{kop},}^{-}, D_{i j k \mathrm{kt}}^{+}\right]$and the real interval allocation of water $A_{i j k \mathrm{kpt}}^{ \pm}=\left[A_{i j k \mathrm{kpt}}^{-}, A_{i j k \mathrm{kpt}}^{+}\right]$can be obtained, while the optimized interval objective value $\bar{f}_{\mathrm{opt}}^{ \pm}=\left[\bar{f}_{\mathrm{opt}}^{-}, \bar{f}_{\mathrm{opt}}^{+}\right]$can be generated through the variation of $D_{i j k o p t}^{ \pm}$.

\section{Case Study}

In this section, the IMSCCP model is applied to the water resources management systems. Just as the statement for 
the hypothetical problem described in Huang and Loucks [30], a water resources manager shoulders the responsibility of delivering water from an unregulated reservoir to three sectors, that is, municipality, industry, and agriculture, during three periods. All users want to know allocated water amount that they can expect over the three periods. If the allocated water satisfies the demand of the user, per unit water will gain net benefit for the local economy. Otherwise, the user will spend more to obtain water from other reservoirs or curtail their expansion plans, which means per unit water deficiency will gain penalty. Table 1 provides the water allocation targets in the three planning periods. Tables 2 and 3 present the distribution of the stream flows in the three periods. Table 4 shows the related economic data. Obviously, the boundaries of the data in Tables 3 and 4 are random variables satisfying different distribution forms. The objective is to obtain $90 \%$-optimistic value to the net system benefit while the constraints of water availability hold with at least a probability of $95 \%$ over the planning horizon.

Based on the information shown in Tables 1-4, the IMSCCP model for water resources management can be formulated as follows:

$$
\begin{aligned}
& \max \bar{f}^{ \pm} \\
& \text {s.t } \operatorname{Pr}\left\{\sum_{i=1}^{3} \sum_{j=1}^{3} \mathrm{NB}_{i j}^{ \pm}\left(T_{i j}^{-}+\Delta T_{i j} y_{i j}\right)-\sum_{i=1}^{3} \sum_{k=1}^{3} p_{1 k} C_{i 1}^{ \pm} D_{i 1 k}^{ \pm}-\sum_{i=1}^{3} \sum_{k=1}^{9} p_{2 k} C_{i 2}^{ \pm} D_{i 2 k}^{ \pm}-\sum_{i=1}^{3} \sum_{k=1}^{27} p_{3 k} C_{i 3}^{ \pm} D_{i 3 k}^{ \pm} \geq \bar{f}^{ \pm}\right\} \geq \alpha \\
& \operatorname{Pr}\left\{\sum_{i=1}^{3}\left(T_{i 1}^{-}+\Delta T_{i 1} y_{i 1}-D_{i 1 k}^{ \pm}\right) \leq q_{1 k}^{ \pm}\right\} \geq \beta_{1 k}, \quad k=1,2,3 \\
& \operatorname{Pr}\left\{\sum_{i=1}^{3}\left(T_{i 2}^{-}+\Delta T_{i 2} y_{i 2}-D_{i 2 k}^{ \pm}\right) \leq q_{2 k}^{ \pm}+\varepsilon_{1 k}^{ \pm}\right\} \geq \beta_{2 k}, \quad k=1,2, \ldots, 9 \\
& \operatorname{Pr}\left\{\sum_{i=1}^{3}\left(T_{i 3}^{-}+\Delta T_{i 3} y_{i 3}-D_{i 3 k}^{ \pm}\right) \leq q_{2 k}^{ \pm}+\varepsilon_{2 k}^{ \pm}\right\} \geq \beta_{3 k}, \quad k=1,2, \ldots, 27 \\
& \varepsilon_{1 k}^{ \pm}=q_{1 k}^{ \pm}-\sum_{i=1}^{3}\left(T_{i 1}^{-}+\Delta T_{i 1} y_{i 1}-D_{i 1}^{ \pm}\right), \quad k=1,2, \ldots, 9 \\
& \varepsilon_{2 k}^{ \pm}=\left[q_{2 k}^{ \pm}-\sum_{i=1}^{3}\left(T_{i 2}^{-}+\Delta T_{i 2} y_{i 2}-D_{i 2}^{ \pm}\right)\right]+\varepsilon_{1 k}^{ \pm}, \quad k=1,2, \ldots, 9 \\
& 0 \leq y_{i j} \leq 1, \quad i=1,2,3, \quad j=1,2,3 \\
& T_{i 1 \max }^{ \pm} \geq T_{i 1}^{-}+\Delta T_{i 1} y_{i 1} \geq D_{i 1 k}^{ \pm} \geq 0, \quad i=1,2,3, \quad k=1,2,3 \\
& T_{i 2 \max }^{ \pm} \geq T_{i 2}^{-}+\Delta T_{i 2} y_{i 2} \geq D_{i 2 k}^{ \pm} \geq 0, \quad i=1,2,3, k=1,2, \ldots, 9 \\
& T_{i 3 \max }^{ \pm} \geq T_{i 3}^{-}+\Delta T_{i 3} y_{i 3} \geq D_{i 3 k}^{ \pm} \geq 0, \quad i=1,2,3, k=1,2, \ldots, 27 .
\end{aligned}
$$

Apparently, model (11) is different from the exiting chance constraint programming models for water resources management which transform the original chance constraints into deterministic equivalents via the theories provided by Charnes et al. [31]. In order to solve this model, a hybrid algorithm which consists of stochastic simulation, BP neural network, and GA is proposed as follows.

Hybrid Algorithm. In order to solve the model, a hybrid algorithm, which incorporates stochastic simulation, back propagation (BP) neural network, and genetic algorithm (GA), is proposed. At first, model (9) and model (10) should be solved by the hybrid algorithm. In the solution process, stochastic simulation is used to generate inputoutput data, BP neural network is used to approximate the functions according to the generated input-output data, and GA is used to enhance the optimization process and obtain a solution to the optimization problem. The flowchart figure of the hybrid algorithm is shown in Figure 1.

Then, the optimized water allocation target and the real allocation of water are achieved. Finally, we obtain the optimal solutions and the optimal value of the model. The concrete step of the algorithm can be summarized as follows. 


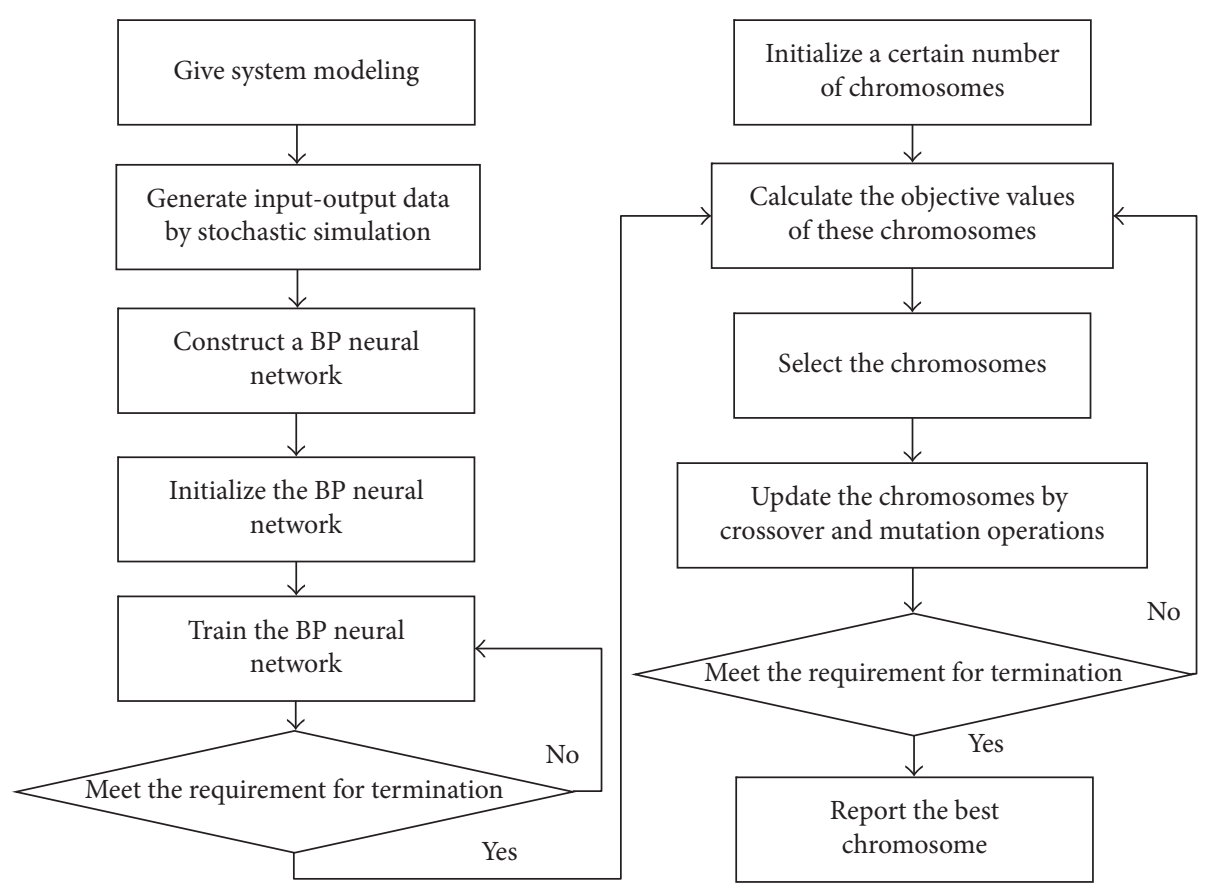

FIGURE 1: The flowchart figure of hybrid algorithm.

TABLE 1: Water allocation targets $\left(10^{6} \mathrm{~m}^{3}\right)$.

\begin{tabular}{lccr}
\hline Water allocation targets & & Time periods & $t=3$ \\
\hline Municipality $(i=1)$ & {$[2,3]$} & $t=2$ & {$[3,4]$} \\
Industrial sector $(i=2)$ & {$[2.5,4]$} & {$[2.5,3.5]$} & {$[4,6]$} \\
Agriculture sector $(i=3)$ & {$[3.5,5.5]$} & {$[3.5,5.5]$} & {$[4,6.5]$} \\
\hline
\end{tabular}

Step 1. Firstly, give system modeling of model (9). Generate a training set of input-output data for the following uncertain functions:

$$
\begin{aligned}
& U_{i j k}^{\prime}\left(y_{i j}, D_{i j k}^{-}\right)=\max \left\{\bar{f}^{+} \mid\right. \\
& \operatorname{Pr}\left\{\sum_{i=1}^{3} \sum_{j=1}^{3} \mathrm{NB}_{i j}^{+}\left(T_{i j}^{-}+\Delta T_{i j} y_{i j}\right)-\sum_{i=1}^{3} \sum_{k=1}^{3} p_{1 k} C_{i 1}^{-} D_{i 1 k}^{-}\right. \\
& \left.-\sum_{i=1}^{3} \sum_{k=1}^{9} p_{2 k} C_{i 2}^{-} D_{i 2 k}^{-}-\sum_{i=1}^{3} \sum_{k=1}^{27} p_{3 k} C_{i 3}^{-} D_{i 3 k}^{-} \geq \bar{f}^{+}\right\} \\
& \geq \alpha\}, \\
& U_{i 1 k}^{\prime \prime}\left(y_{i 1}, D_{i 1 k}^{-}\right)=\operatorname{Pr}\left\{\sum_{i=1}^{3}\left(T_{i 1}^{-}+\Delta T_{i 1} y_{i 1}-D_{i 1 k}^{-}\right)\right. \\
& \left.\leq q_{1 k}^{+}\right\}, \quad k=1,2,3,
\end{aligned}
$$

$$
\begin{aligned}
& U_{i 2 k}^{\prime \prime}\left(y_{i 2}, D_{i 2 k}^{-}\right)=\operatorname{Pr}\left\{\sum_{i=1}^{3}\left(T_{i 2}^{-}+\Delta T_{i 2} y_{i 2}-D_{i 2 k}^{-}\right) \leq q_{2 k}^{+}\right. \\
& \left.+\varepsilon_{1 k}^{+}\right\}, \quad k=1,2, \ldots, 9, \\
& U_{i 3 k}^{\prime \prime}\left(y_{i 3}, D_{i 3 k}^{-}\right)=\operatorname{Pr}\left\{\sum_{i=1}^{3}\left(T_{i 3}^{-}+\Delta T_{i 3} y_{i 3}-D_{i 3 k}^{-}\right) \leq q_{2 k}^{+}\right. \\
& \left.+\varepsilon_{2 k}^{+}\right\}, \quad k=1,2, \ldots, 27
\end{aligned}
$$

by stochastic simulation (the basic principle could be seen in Liu [21]).

Secondly, construct a BP neural network to approximate the functions according to the generated input-output data and then initialize and train the BP neural network.

Thirdly, initialize a certain number of chromosomes according to the distribution function and check the feasibility of these chromosomes.

Fourthly, calculate the values of the objective function as fitness value by the trained BP neural network. 
TABLE 2: Seasonal flows in the three planning periods $\left(10^{6} \mathrm{~m}^{3}\right)$.

\begin{tabular}{lccc}
\hline Time periods & \multicolumn{3}{c}{ Seasonal flows } \\
Low flow $(j=1)$ & Medium flow $(j=2)$ & High flow $(j=3)$ \\
\hline$t=1$ & {$[3.5,4.5]$} & {$[6,8]$} & {$[12,15]$} \\
$t=2$ & {$[5,6]$} & {$[7,10]$} & {$[13,17]$} \\
$t=3$ & {$[3.5,4.5]$} & {$[7.5,11]$} & {$[13.5,17.5]$} \\
\hline Probability & 0.2 & 0.6 & 0.2 \\
\hline
\end{tabular}

TABLE 3: The distribution forms of the boundaries of seasonal flows in the three planning periods $\left(10^{6} \mathrm{~m}^{3}\right)$.

\begin{tabular}{lccc}
\hline Time periods & Low flow $(j=1)$ & $\begin{array}{c}\text { Seasonal flows } \\
\text { Medium flow }(j=2)\end{array}$ & High flow $(j=3)$ \\
\hline$t=1$ & {$\left[N\left(3.5,0.1^{2}\right), N\left(4.5,0.1^{2}\right)\right]$} & {$\left[N\left(6,0.1^{2}\right), N\left(8,0.1^{2}\right)\right]$} & {$\left[N\left(12,0.1^{2}\right), N\left(15,0.1^{2}\right)\right]$} \\
$t=2$ & {$\left[N\left(5,0.1^{2}\right), N\left(6,0.1^{2}\right)\right]$} & {$\left[N\left(7,0.1^{2}\right), N\left(10,0.1^{2}\right)\right]$} & {$\left[N\left(13,0.1^{2}\right), N\left(17,0.1^{2}\right)\right]$} \\
$t=3$ & {$\left[N\left(3.5,0.1^{2}\right), N\left(4.5,0.1^{2}\right)\right]$} & {$\left[N\left(7.5,0.1^{2}\right), N\left(11,0.1^{2}\right)\right]$} & {$\left[N\left(13.5,0.1^{2}\right), N\left(17.5,0.1^{2}\right)\right]$} \\
\hline
\end{tabular}

Note. $N\left(\mu, \sigma^{2}\right)$ represents a normally distributed random variable with mean $\mu$ and standard deviation $\sigma$.

Fifthly, select the chromosomes by running a standard scheme of the roulette wheel.

Sixthly, update the chromosomes by crossover and mutation operations, test the feasibility of chromosome offspring, and obtain a feasible new population.

Seventhly, choose the chromosome which has the maximal fitness while the function values of $U_{i j k}^{\prime \prime}\left(y_{i 1}, D_{i j k}^{-}\right)$are greater than $\beta_{j k}$.

Eighthly, repeat substep 5 to substep 7 for a given number of cycles.

Ninthly, report the best chromosome. The best solutions obtained in the above way are denoted by $D_{i j k \mathrm{opt}}^{-}$and $y_{i j \mathrm{opt}}$, and the optimal value is denoted by $\bar{f}_{\text {opt }}^{+}$.

Step 2. Based on the optimal solutions obtained by Step 1, system modeling of model (10) is given. Generate a training set of input-output data for the following uncertain functions:

$$
\begin{aligned}
& G_{i j k}^{\prime}\left(D_{i j k}^{+}\right)=\max \left\{\bar{f}^{-} \mid\right. \\
& \operatorname{Pr}\left\{\sum_{i=1}^{3} \sum_{j=1}^{3} \mathrm{NB}_{i j}^{-}\left(T_{i j}^{-}+\Delta T_{i j} y_{i j \mathrm{opt}}\right)\right. \\
& -\sum_{i=1}^{3} \sum_{k=1}^{3} p_{1 k} C_{i 1}^{+} D_{i 1 k}^{+}-\sum_{i=1}^{3} \sum_{k=1}^{9} p_{2 k} C_{i 2}^{+} D_{i 2 k}^{+} \\
& \left.\left.-\sum_{i=1}^{3} \sum_{k=1}^{27} p_{3 k} C_{i 3}^{+} D_{i 3 k}^{+} \geq \bar{f}\right\} \geq \alpha\right\}, \\
& G_{i 1 k}^{\prime \prime}\left(D_{i 1 k}^{+}\right)=\operatorname{Pr}\left\{\sum_{i=1}^{3}\left(T_{i 1}^{-}+\Delta T_{i 1} y_{i 1 \mathrm{opt}}-D_{i 1 k}^{+}\right) \leq q_{1 k}^{-}\right\} \\
& k=1,2,3,
\end{aligned}
$$

$$
\begin{aligned}
& G_{i 2 k}^{\prime \prime}\left(D_{i 2 k}^{+}\right)=\operatorname{Pr}\left\{\sum_{i=1}^{3}\left(T_{i 2}^{-}+\Delta T_{i 2} y_{i 2 \mathrm{opt}}-D_{i 2 k}^{+}\right) \leq q_{2 k}^{-}\right. \\
& \left.+\varepsilon_{1 k}^{-}\right\}, \quad k=1,2, \ldots, 9, \\
& G_{i 3 k}^{\prime \prime}\left(D_{i 3 k}^{+}\right)=\operatorname{Pr}\left\{\sum_{i=1}^{3}\left(T_{i 3}^{-}+\Delta T_{i 3} y_{i 3 \mathrm{opt}}-D_{i 3 k}^{+}\right) \leq q_{2 k}^{-}\right. \\
& \left.+\varepsilon_{2 k}^{-}\right\}, \quad k=1,2, \ldots, 27
\end{aligned}
$$

by stochastic simulation. The remaining substeps are similar to the substeps in Step 1. Finally, report the best chromosome. The optimal solution obtained in the above way is $D_{i j k o p t}^{+}$and the optimal value is $\bar{f}_{\text {opt }}$.

Step 3. Achieve the optimized water allocation target $T_{i j \text { opt }}^{ \pm}=$ $T_{i j}^{-}+\Delta T_{i j} y_{i j o p t}$ and the real allocation of water $A_{i j k \mathrm{opt}}^{ \pm}=T_{i j \mathrm{opt}}^{ \pm}-$ $D_{i j k o p t}^{ \pm}$.

Step 4. Synthesize the two submodels. The optimal solutions can be summarized as $D_{i j k \mathrm{opt}}^{ \pm}=\left[D_{i j k \mathrm{opt}}^{-}, D_{i j k \mathrm{opt}}^{+}\right]$and the optimal value can be summarized as $\bar{f}_{\mathrm{opt}}^{ \pm}=\left[\bar{f}_{\mathrm{opt}}, \bar{f}_{\mathrm{opt}}^{+}\right]$.

\section{Results and Discussions}

The demands and deficits for water are related to water availability, since the economic benefit will be obtained if the demands are satisfied, while the economic penalties will be generated if the deficits occur. Table 1 provides water allocation targets in the three planning periods. Tables 2 and 3 present the information regarding seasonal flows under different probabilities. Tables 1-3 derive from [1]. In the case of insufficient water resources, the water for municipal sector should be delivered preferentially since the highest 
TABLE 4: Net benefit and penalty $\left(\$ / \mathrm{m}^{3}\right)$.

\begin{tabular}{lccc}
\hline & & Time period \\
& $t=1$ & $t=2$ & $t=3$ \\
\hline $\begin{array}{l}\text { Net benefit when water demand is satisfied } \\
\text { Municipality }\end{array}$ & {$[U(90,91), U(110,111)]$} & {$[U(95,96), U(115,116)]$} & {$[U(100,101), U(130,131)]$} \\
$\quad \begin{array}{l}\text { Industrial sector } \\
\text { Agricultural sector }\end{array}$ & {$\left[N\left(45,0.1^{2}\right), N\left(50,0.1^{2}\right)\right]$} & {$\left[N\left(50,0.1^{2}\right), N\left(60,0.1^{2}\right)\right]$} & {$\left[N\left(55,0.1^{2}\right), N\left(70,0.1^{2}\right)\right]$} \\
\hline Penalty when water is not delivered & {$\left[N\left(30,0.1^{2}\right), N\left(35,0.1^{2}\right)\right]$} & {$\left[N\left(35,0.1^{2}\right), N\left(42,0.1^{2}\right)\right]$} & {$\left[N\left(40,0.1^{2}\right), N\left(58,0.1^{2}\right)\right]$} \\
$\quad$ Municipality & {$[U(200,201), U(250,251)]$} & {$[U(220,221), U(275,276)]$} & {$[U(240,241), U(300,301)]$} \\
Industrial sector & {$\left[N\left(60,0.1^{2}\right), N\left(85,0.1^{2}\right)\right]$} & {$\left[N\left(70,0.1^{2}\right), N\left(95,0.1^{2}\right)\right]$} & {$\left[N\left(80,0.1^{2}\right), N\left(110,0.1^{2}\right)\right]$} \\
Agricultural sector & {$\left[N\left(50,0.1^{2}\right), N\left(70,0.1^{2}\right)\right]$} & {$\left[N\left(55,0.1^{2}\right), N\left(75,0.1^{2}\right)\right]$} & {$\left[N\left(60,0.1^{2}\right), N\left(90,0.1^{2}\right)\right]$} \\
\hline
\end{tabular}

Note. $U(a, b)$ represents a uniform distributed random variable and $N\left(\mu, \sigma^{2}\right)$ represents a normally distributed random variable.

TABLE 5: Solutions for the first planning period $(\alpha=0.9, \beta=0.95)$.

\begin{tabular}{|c|c|c|c|c|c|c|}
\hline Scenario symbol $(i j k)$ & User & Water flow level & Probability (\%) & Water targets $\left(10^{6} \mathrm{~m}^{3}\right)$ & Water shortage $\left(10^{6} \mathrm{~m}^{3}\right)$ & $\begin{array}{l}\text { Water allocation } \\
\qquad\left(10^{6} \mathrm{~m}^{3}\right)\end{array}$ \\
\hline 111 & Municipal & $\mathrm{L}$ & 20 & 2.644 & {$[1.105,1.174]$} & {$[1.470,1.539]$} \\
\hline 211 & Industrial & $\mathrm{L}$ & 20 & 3.615 & {$[3.342,3.353]$} & {$[0.262,0.273]$} \\
\hline 311 & Agricultural & $\mathrm{L}$ & 20 & 4.000 & {$[3.524,3.769]$} & {$[0.231,0.476]$} \\
\hline 112 & Municipal & M & 60 & 2.644 & {$[2.116,2.348]$} & {$[0.296,0.528]$} \\
\hline 212 & Industrial & M & 60 & 3.615 & {$[2.469,2.531]$} & {$[1.084,1.146]$} \\
\hline 312 & Agricultural & M & 60 & 4.000 & {$[0.889,2.132]$} & {$[1.868,3.111]$} \\
\hline 113 & Municipal & $\mathrm{H}$ & 20 & 2.644 & {$[1.200,1.328]$} & {$[1.316,1.444]$} \\
\hline 213 & Industrial & $\mathrm{H}$ & 20 & 3.615 & {$[2.467,3.159]$} & {$[0.456,1.148]$} \\
\hline 313 & Agricultural & $\mathrm{H}$ & 20 & 4.000 & {$[2.239,3.411]$} & {$[0.589,1.761]$} \\
\hline
\end{tabular}

$y_{11 \mathrm{opt}}=0.644, y_{21 \mathrm{opt}}=0.743$, and $y_{31 \mathrm{opt}}=0.250$.

benefit will be brought when the municipal water demand is satisfied, while the highest penalty will be produced if the promised water is not delivered, followed by the industrial and agricultural sectors which correspond to lower benefits and penalties (see Table 4).

The solutions shown in Tables 5-7 ensure that the 90\% optimistic value to the net system benefit could be obtained subject to the chance constraints with a confidence level of $95 \%$. Therefore, the confidence levels for the net system benefit and the chance constraints during the three planning periods are set to $\alpha=0.9$ and $\beta_{j k}=0.95$ for $k=1,2, \ldots, K_{j}$, $j=1,2,3$. In solution process by using hybrid algorithm, the maximum number of iterations, the learning rate, the momentum term, and the tolerance criterion for the BP neural network are set to be 20000, 0.01, 0.9, and 0.00001, respectively. The population size, the number of generations, the mutation rate, and the crossover rate of GA are set to be $30,300,0.1$, and 0.7 , respectively. The solutions indicate that $y_{11 \mathrm{opt}}=0.644, y_{21 \mathrm{opt}}=0.743, y_{31 \mathrm{opt}}=0.250, y_{12 \mathrm{opt}}=0.449$, $y_{22 \mathrm{opt}}=0.281, y_{31 \mathrm{opt}}=0.182, y_{13 \mathrm{opt}}=0.212, y_{23 \mathrm{opt}}=0.211$ and $y_{33 \mathrm{opt}}=0.375$. Thus, the optimized allocated targets are $T_{11 \mathrm{opt}}^{ \pm}=2.644, T_{21 \mathrm{opt}}^{ \pm}=3.615, T_{31 \mathrm{opt}}^{ \pm}=4.000, T_{12 \mathrm{opt}}^{ \pm}=2.949$, $T_{22 \mathrm{opt}}^{ \pm}=4.062, T_{32 \mathrm{opt}}^{ \pm}=4.363, T_{13 \mathrm{opt}}^{ \pm}=3.212, T_{23 \mathrm{opt}}^{ \pm}=4.423$, and $T_{33 \text { opt }}^{ \pm}=4.938$. These targets would be promised to the three users in the first stage. Obviously, the water manager's decisions should balance the net benefit and the risk because high net benefit would be brought if the promised water is delivered and high penalties would occur if the demand for water is not achieved.

Table 5 indicates the optimized solutions under 3 scenarios for the first planning period. For example, $D_{111 \mathrm{opt}}^{ \pm}=$ $[1.105,1.174], D_{112 \mathrm{opt}}^{ \pm}=[2.116,2.348]$, and $D_{113 \mathrm{opt}}^{ \pm}=$ $[1.200,1.328]$ are water shortages for the municipal sector $(j=1)$ when the water flow level is low, medium, and high with probability of $20 \%, 60 \%$, and $20 \%$, respectively. Accordingly, the water allocations are $A_{111 \mathrm{opt}}^{ \pm}=[1.470,1.539]$, $A_{112 \mathrm{opt}}^{ \pm}=[0.296,0.528]$, and $A_{113 \mathrm{opt}}^{ \pm}=[1.316,1.444]$.

Table 6 indicates the optimized solutions under 9 scenarios for the second planning period. Take water shortages and water allocations of the industrial sector: for example, $(j=2) . D_{224 \text { opt }}^{ \pm}=[1.940,3.328], D_{225 \text { opt }}^{ \pm}=[1.566,2.654]$, and $D_{226 \text { opt }}^{ \pm}=[3.111,3.642]$ denote water shortages for the industrial sector when the water flow level in the second period is low, medium, and high following the medium flow in the previous period with joint probability of $12 \%$, $36 \%$, and $12 \%$, respectively. Accordingly, the water allocations are $A_{224 \text { opt }}^{ \pm}=[0.734,2.122], A_{225 \text { opt }}^{ \pm}=[1.408,2.496]$, and $A_{226 \text { opt }}^{ \pm}=[0.420,0.951]$.

Table 7 provides optimized water allocation schemes under 27 scenarios for the third planning period. For example, $D_{1322 \text { opt }}^{ \pm}=[2.553,2.817], D_{2322 \mathrm{opt}}^{ \pm}=[4.047,4.256]$, 
TABLE 6: Solutions for the second planning period $(\alpha=0.9, \beta=0.95)$.

\begin{tabular}{|c|c|c|c|c|c|c|c|c|}
\hline $\begin{array}{l}\text { Scenario } \\
\text { symbol } \\
(i j k) \\
\end{array}$ & User & $\begin{array}{c}\text { Water flow } \\
\text { level }\end{array}$ & $\begin{array}{l}\text { Probability } \\
\text { (\%) }\end{array}$ & $\begin{array}{l}\text { Associated } \\
\text { water flow }\end{array}$ & $\begin{array}{c}\text { Associated } \\
\text { probability } \\
(\%)\end{array}$ & $\begin{array}{l}\text { Water target } \\
\qquad\left(10^{6} \mathrm{~m}^{3}\right)\end{array}$ & $\begin{array}{l}\text { Water shortage } \\
\qquad\left(10^{6} \mathrm{~m}^{3}\right)\end{array}$ & $\begin{array}{l}\text { Water allocation } \\
\qquad\left(10^{6} \mathrm{~m}^{3}\right)\end{array}$ \\
\hline 121 & Municipal & $\mathrm{L}$ & 20 & L-L & 4 & 2.949 & {$[0.899,1.683]$} & {$[1.266,2.050]$} \\
\hline 221 & Industrial & $\mathrm{L}$ & 20 & $\mathrm{~L}-\mathrm{L}$ & 4 & 4.062 & {$[0.050,0.764]$} & {$[3.298,4.012]$} \\
\hline 321 & Agricultural & $\mathrm{L}$ & 20 & $\mathrm{~L}-\mathrm{L}$ & 4 & 4.363 & {$[3.032,3.452]$} & {$[0.911,1.331]$} \\
\hline 122 & Municipal & M & 60 & L-M & 12 & 2.949 & {$[0.369,2.649]$} & {$[0.300,2.580]$} \\
\hline 222 & Industrial & M & 60 & L-M & 12 & 4.062 & {$[1.641,3.174]$} & {$[0.888,2.421]$} \\
\hline 322 & Agricultural & M & 60 & L-M & 12 & 4.363 & {$[3.968,4.155]$} & {$[0.208,0.395]$} \\
\hline 123 & Municipal & $\mathrm{H}$ & 20 & L-H & 4 & 2.949 & {$[0.710,1.032]$} & {$[1.917,2.239]$} \\
\hline 223 & Industrial & $\mathrm{H}$ & 20 & $\mathrm{~L}-\mathrm{H}$ & 4 & 4.062 & {$[0.408,1.328]$} & {$[2.734,3.654]$} \\
\hline 323 & Agricultural & $\mathrm{H}$ & 20 & L-H & 4 & 4.363 & {$[1.334,3.588]$} & {$[0.775,3.029]$} \\
\hline 124 & Municipal & $\mathrm{L}$ & 20 & M-L & 12 & 2.949 & {$[2.910,2.931]$} & {$[0.018,0.039]$} \\
\hline 224 & Industrial & $\mathrm{L}$ & 20 & M-L & 12 & 4.062 & {$[1.940,3.328]$} & {$[0.734,2.122]$} \\
\hline 324 & Agricultural & $\mathrm{L}$ & 20 & M-L & 12 & 4.363 & {$[3.400,3.948]$} & {$[0.415,0.963]$} \\
\hline 125 & Municipal & M & 60 & M-M & 36 & 2.949 & {$[2.941,2.947]$} & {$[0.002,0.008]$} \\
\hline 225 & Industrial & M & 60 & M-M & 36 & 4.062 & {$[1.566,2.654]$} & {$[1.408,2.496]$} \\
\hline 325 & Agricultural & M & 60 & M-M & 36 & 4.363 & {$[0.159,3.291]$} & {$[1.072,4.204]$} \\
\hline 126 & Municipal & $\mathrm{H}$ & 20 & $\mathrm{M}-\mathrm{H}$ & 12 & 2.949 & {$[2.381,2.774]$} & {$[0.175,0.568]$} \\
\hline 226 & Industrial & $\mathrm{H}$ & 20 & M-H & 12 & 4.062 & {$[3.111,3.642]$} & {$[0.420,0.951]$} \\
\hline 326 & Agricultural & $\mathrm{H}$ & 20 & $\mathrm{M}-\mathrm{H}$ & 12 & 4.363 & {$[1.318,3.148]$} & {$[1.215,3.045]$} \\
\hline 127 & Municipal & $\mathrm{L}$ & 20 & $\mathrm{H}-\mathrm{L}$ & 4 & 2.949 & {$[2.534,2.669]$} & {$[0.280,0.415]$} \\
\hline 227 & Industrial & $\mathrm{L}$ & 20 & $\mathrm{H}-\mathrm{L}$ & 4 & 4.062 & {$[1.557,1.709]$} & {$[2.353,2.505]$} \\
\hline 327 & Agricultural & $\mathrm{L}$ & 20 & $\mathrm{H}-\mathrm{L}$ & 4 & 4.363 & {$[3.310,4.033]$} & {$[0.330,1.053]$} \\
\hline 128 & Municipal & M & 60 & $\mathrm{H}-\mathrm{M}$ & 12 & 2.949 & {$[2.755,2.821]$} & {$[0.128,0.194]$} \\
\hline 228 & Industrial & M & 60 & $\mathrm{H}-\mathrm{M}$ & 12 & 4.062 & {$[3.088,3.617]$} & {$[0.445,0.974]$} \\
\hline 328 & Agricultural & M & 60 & $\mathrm{H}-\mathrm{M}$ & 12 & 4.363 & {$[0.374,0.883]$} & {$[3.480,3.989]$} \\
\hline 129 & Municipal & $\mathrm{H}$ & 20 & $\mathrm{H}-\mathrm{H}$ & 4 & 2.949 & {$[0.557,1.088]$} & {$[1.861,2.392]$} \\
\hline 229 & Industrial & $\mathrm{H}$ & 20 & $\mathrm{H}-\mathrm{H}$ & 4 & 4.062 & {$[0.678,2.590]$} & {$[1.472,3.384]$} \\
\hline 329 & Agricultural & $\mathrm{H}$ & 20 & $\mathrm{H}-\mathrm{H}$ & 4 & 4.363 & {$[0.402,3.609]$} & {$[0.754,3.961]$} \\
\hline
\end{tabular}

$y_{12 \mathrm{opt}}=0.449, y_{22 \mathrm{opt}}=0.281$, and $y_{31 \mathrm{opt}}=0.182$.

and $D_{3322 \text { opt }}^{ \pm}=[0.542,4.078]$ indicate the water shortages for industrial, agricultural, and municipal sectors with the corresponding water allocations $A_{1333 \mathrm{opt}}^{ \pm}=[0.395,0.659]$, $A_{2322 \mathrm{opt}}^{ \pm}=[0.167,0.376]$, and $A_{3322 \mathrm{opt}}^{ \pm}=[0.860,4.396]$ respectively, when the water flows are high-medium-low during the entire planning horizon with joint probability of $2.4 \%$.

The optimal value $\left(\bar{f}_{\text {opt }}^{ \pm}=[154.335,235.704]\right)$ represents the $90 \%$ optimistic value to the net system benefit subject to the chance constraints with a confidence level of $95 \%$, which provides two extreme values over the planning horizon. Planning for a lower system benefit would be associated with a lower risk of violating the water allocation constraints; conversely, the desire for a higher benefit would correspond to a higher possibility of violating the constraints [9]. When the actual value of each variable fluctuates between its lower and upper bounds, the $90 \%$ optimistic values to the net system benefit would change correspondingly between
154.335 and 235.704, which reflects the balance between the system profit and the chance constraints.

In the hypothetical case, the water resource is an unregulated reservoir and the uncertainties in the water resources management are expressed as interval with uniform distributed and normally distributed boundaries. However, realworld water resources systems are more complex than the hypothetical case presented, since the water allocated to the users is from regulated reservoir(s), and the representation of the uncertainties in the input may be more diversified. Nevertheless, the hypothetical case still reflects the basic scene and principles and contains main information in the real water resources management systems. Simple as it is, the case is sufficient to study the characteristics and optimization problems of real-world water resources systems. For the real water resources management problems which contain data with multiply distributed (e.g., Gamma, Lognormal) stochastic boundaries, they can also be dealt with through establishing the appropriate IMSCCP model and adding 
TABLE 7: Solutions for the third planning period $(\alpha=0.9, \beta=0.95)$.

\begin{tabular}{|c|c|c|c|c|c|c|c|c|}
\hline $\begin{array}{l}\text { Scenario } \\
\text { symbol } \\
(i j k)\end{array}$ & User & $\begin{array}{c}\text { Water flow } \\
\text { level }\end{array}$ & $\begin{array}{c}\text { Probability } \\
\text { (\%) }\end{array}$ & $\begin{array}{l}\text { Associated } \\
\text { water flow }\end{array}$ & $\begin{array}{l}\text { Associated } \\
\text { probability } \\
(\%)\end{array}$ & $\begin{array}{l}\text { Water targets } \\
\qquad\left(10^{6} \mathrm{~m}^{3}\right)\end{array}$ & $\begin{array}{l}\text { Water shortage } \\
\qquad\left(10^{6} \mathrm{~m}^{3}\right)\end{array}$ & $\begin{array}{l}\text { Water allocation } \\
\qquad\left(10^{6} \mathrm{~m}^{3}\right)\end{array}$ \\
\hline 131 & Municipal & $\mathrm{L}$ & 20 & L-L-L & 0.8 & 3.212 & {$[2.543,2.696]$} & {$[0.516,0.669]$} \\
\hline 231 & Industrial & $\mathrm{L}$ & 20 & L-L-L & 0.8 & 4.423 & {$[1.114,3.435]$} & {$[0.988,3.309]$} \\
\hline 331 & Agricultural & $\mathrm{L}$ & 20 & L-L-L & 0.8 & 4.938 & {$[4.929,4.935]$} & {$[0.003,0.009]$} \\
\hline 132 & Municipal & M & 60 & L-L-M & 2.4 & 3.212 & {$[0.410,1.275]$} & {$[1.937,2.802]$} \\
\hline 232 & Industrial & M & 60 & L-L-M & 2.4 & 4.423 & {$[1.376,3.065]$} & {$[1.358,3.047]$} \\
\hline 332 & Agricultural & M & 60 & L-L-M & 2.4 & 4.938 & {$[3.665,4.086]$} & {$[0.852,1.273]$} \\
\hline 133 & Municipal & $\mathrm{H}$ & 20 & L-L-H & 0.8 & 3.212 & {$[1.450,1.573]$} & {$[1.639,1.762]$} \\
\hline 233 & Industrial & $\mathrm{H}$ & 20 & L-L-H & 0.8 & 4.423 & {$[0.144,4.207]$} & {$[0.216,4.279]$} \\
\hline 333 & Agricultural & $\mathrm{H}$ & 20 & L-L-H & 0.8 & 4.938 & {$[3.899,4.276]$} & {$[0.662,1.039]$} \\
\hline 134 & Municipal & $\mathrm{L}$ & 20 & L-M-L & 2.4 & 3.212 & {$[2.658,2.876]$} & {$[0.336,0.554]$} \\
\hline 234 & Industrial & $\mathrm{L}$ & 20 & L-M-L & 2.4 & 4.423 & {$[2.895,3.297]$} & {$[1.126,1.528]$} \\
\hline 334 & Agricultural & $\mathrm{L}$ & 20 & L-M-L & 2.4 & 4.938 & {$[1.394,4.583]$} & {$[0.355,3.544]$} \\
\hline 135 & Municipal & M & 60 & L-M-M & 7.2 & 3.212 & {$[3.171,3.199]$} & {$[0.013,0.041]$} \\
\hline 235 & Industrial & $\mathrm{M}$ & 60 & L-M-M & 7.2 & 4.423 & {$[0.876,3.090]$} & {$[1.333,3.547]$} \\
\hline 335 & Agricultural & M & 60 & L-M-M & 7.2 & 4.938 & {$[0.782,2.813]$} & {$[2.125,4.156]$} \\
\hline 136 & Municipal & $\mathrm{H}$ & 20 & L-M-H & 2.4 & 3.212 & {$[2.023,2.385]$} & {$[0.827,1.189]$} \\
\hline 236 & Industrial & $\mathrm{H}$ & 20 & L-M-H & 2.4 & 4.423 & {$[2.709,3.584]$} & {$[0.839,1.714]$} \\
\hline 336 & Agricultural & $\mathrm{H}$ & 20 & L-M-H & 2.4 & 4.938 & {$[2.682,3.085]$} & {$[1.853,2.256]$} \\
\hline 137 & Municipal & $\mathrm{L}$ & 20 & L-H-L & 0.8 & 3.212 & {$[2.037,3.022]$} & {$[0.190,1.175]$} \\
\hline 237 & Industrial & $\mathrm{L}$ & 20 & L-H-L & 0.8 & 4.423 & {$[2.642,3.314]$} & {$[1.109,1.781]$} \\
\hline 337 & Agricultural & $\mathrm{L}$ & 20 & L-H-L & 0.8 & 4.938 & {$[1.762,2.322]$} & {$[2.616,3.176]$} \\
\hline 138 & Municipal & M & 60 & L-H-M & 2.4 & 3.212 & {$[0.929,2.366]$} & {$[0.846,2.283]$} \\
\hline 238 & Industrial & M & 60 & L-H-M & 2.4 & 4.423 & {$[1.283,1.894]$} & {$[2.529,3.140]$} \\
\hline 338 & Agricultural & $\mathrm{M}$ & 60 & L-H-M & 2.4 & 4.938 & {$[3.712,4.480]$} & {$[0.458,1.226]$} \\
\hline 139 & Municipal & $\mathrm{H}$ & 20 & L-H-H & 0.8 & 3.212 & {$[2.564,3.084]$} & {$[0.128,0.648]$} \\
\hline 239 & Industrial & $\mathrm{H}$ & 20 & L-H-H & 0.8 & 4.423 & {$[4.098,4.263]$} & {$[0.160,0.325]$} \\
\hline 339 & Agricultural & $\mathrm{H}$ & 20 & L-H-H & 0.8 & 4.938 & {$[3.183,4.010]$} & {$[0.928,1.755]$} \\
\hline 1310 & Municipal & $\mathrm{L}$ & 20 & M-L-L & 2.4 & 3.212 & {$[1.780,2.296]$} & {$[0.916,1.432]$} \\
\hline 2310 & Industrial & $\mathrm{L}$ & 20 & M-L-L & 2.4 & 4.423 & {$[1.078,3.080]$} & {$[1.343,3.345]$} \\
\hline 3310 & Agricultural & $\mathrm{L}$ & 20 & M-L-L & 2.4 & 4.938 & {$[4.624,4.856]$} & {$[0.082,0.314]$} \\
\hline 1311 & Municipal & M & 60 & M-L-M & 7.2 & 3.212 & {$[1.169,3.032]$} & {$[0.180,2.043]$} \\
\hline 2311 & Industrial & $\mathrm{M}$ & 60 & M-L-M & 7.2 & 4.423 & {$[2.145,3.887]$} & {$[0.536,2.278]$} \\
\hline 3311 & Agricultural & M & 60 & M-L-M & 7.2 & 4.938 & {$[3.619,4.577]$} & {$[0.361,1.319]$} \\
\hline 1312 & Municipal & $\mathrm{H}$ & 20 & M-L-H & 2.4 & 3.212 & {$[1.509,2.804]$} & {$[0.408,1.703]$} \\
\hline 2312 & Industrial & $\mathrm{H}$ & 20 & M-L-H & 2.4 & 4.423 & {$[0.905,2.582]$} & {$[1.841,3.518]$} \\
\hline 3312 & Agricultural & $\mathrm{H}$ & 20 & M-L-H & 2.4 & 4.938 & {$[2.117,2.380]$} & {$[2.558,2.821]$} \\
\hline 1313 & Municipal & $\mathrm{L}$ & 20 & M-M-L & 7.2 & 3.212 & {$[0.628,2.264]$} & {$[0.948,2.584]$} \\
\hline 2313 & Industrial & $\mathrm{L}$ & 20 & M-M-L & 7.2 & 4.423 & {$[2.505,2.865]$} & {$[1.558,1.918]$} \\
\hline 3313 & Agricultural & $\mathrm{L}$ & 20 & M-M-L & 7.2 & 4.938 & {$[4.341,4.492]$} & {$[0.446,0.597]$} \\
\hline 1314 & Municipal & M & 60 & M-M-M & 21.6 & 3.212 & {$[1.192,3.049]$} & {$[0.163,2.020]$} \\
\hline 2314 & Industrial & $\mathrm{M}$ & 60 & M-M-M & 21.6 & 4.423 & {$[1.062,2.988]$} & {$[1.435,3.361]$} \\
\hline 3314 & Agricultural & M & 60 & M-M-M & 21.6 & 4.938 & {$[1.389,3.794]$} & {$[1.144,3.549]$} \\
\hline 1315 & Municipal & $\mathrm{H}$ & 20 & M-M-H & 7.2 & 3.212 & {$[1.946,2.103]$} & {$[1.109,1.266]$} \\
\hline 2315 & Industrial & $\mathrm{H}$ & 20 & M-M-H & 7.2 & 4.423 & {$[1.948,3.230]$} & {$[1.193,2.475]$} \\
\hline 3315 & Agricultural & $\mathrm{H}$ & 20 & M-M-H & 7.2 & 4.938 & {$[2.309,2.817]$} & {$[2.121,2.629]$} \\
\hline 1316 & Municipal & $\mathrm{L}$ & 20 & M-H-L & 2.4 & 3.212 & {$[1.733,2.390]$} & {$[0.822,1.479]$} \\
\hline
\end{tabular}


TABle 7: Continued.

\begin{tabular}{|c|c|c|c|c|c|c|c|c|}
\hline $\begin{array}{l}\text { Scenario } \\
\text { symbol } \\
(i j k)\end{array}$ & User & $\begin{array}{l}\text { Water flow } \\
\text { level }\end{array}$ & $\begin{array}{l}\text { Probability } \\
\text { (\%) }\end{array}$ & $\begin{array}{l}\text { Associated } \\
\text { water flow }\end{array}$ & $\begin{array}{c}\text { Associated } \\
\text { probability } \\
(\%)\end{array}$ & $\begin{array}{l}\text { Water targets } \\
\qquad\left(10^{6} \mathrm{~m}^{3}\right)\end{array}$ & $\begin{array}{l}\text { Water shortage } \\
\qquad\left(10^{6} \mathrm{~m}^{3}\right)\end{array}$ & $\begin{array}{l}\text { Water allocation } \\
\qquad\left(10^{6} \mathrm{~m}^{3}\right)\end{array}$ \\
\hline 2316 & Industrial & $\mathrm{L}$ & 20 & M-H-L & 2.4 & 4.423 & {$[1.409,4.280]$} & {$[0.143,3.014]$} \\
\hline 3316 & Agricultural & $\mathrm{L}$ & 20 & M-H-L & 2.4 & 4.938 & {$[4.207,4.583]$} & {$[0.355,0.731]$} \\
\hline 1317 & Municipal & M & 60 & M-H-M & 7.2 & 3.212 & {$[2.431,2.657]$} & {$[0.555,0.781]$} \\
\hline 2317 & Industrial & M & 60 & M-H-M & 7.2 & 4.423 & {$[1.526,2.959]$} & {$[1.464,2.897]$} \\
\hline 3317 & Agricultural & M & 60 & M-H-M & 7.2 & 4.938 & {$[1.660,3.432]$} & {$[1.506,3.278]$} \\
\hline 1318 & Municipal & $\mathrm{H}$ & 20 & M-H-H & 2.4 & 3.212 & {$[0.980,1.552]$} & {$[1.660,2.232]$} \\
\hline 2318 & Industrial & $\mathrm{H}$ & 20 & $\mathrm{M}-\mathrm{H}-\mathrm{H}$ & 2.4 & 4.423 & {$[2.041,3.262]$} & {$[1.161,2.382]$} \\
\hline 3318 & Agricultural & $\mathrm{H}$ & 20 & M-H-H & 2.4 & 4.938 & {$[2.407,3.482]$} & {$[1.456,2.531]$} \\
\hline 1319 & Municipal & $\mathrm{L}$ & 20 & H-L-L & 0.8 & 3.212 & {$[0.145,1.877]$} & {$[1.335,3.067]$} \\
\hline 2319 & Industrial & $\mathrm{L}$ & 20 & H-L-L & 0.8 & 4.423 & {$[1.486,2.048]$} & {$[2.375,2.937]$} \\
\hline 3319 & Agricultural & $\mathrm{L}$ & 20 & H-L-L & 0.8 & 4.938 & {$[3.269,4.521]$} & {$[0.417,1.669]$} \\
\hline 1320 & Municipal & M & 60 & H-L-M & 2.4 & 3.212 & {$[1.494,2.802]$} & {$[0.410,1.718]$} \\
\hline 2320 & Industrial & M & 60 & H-L-M & 2.4 & 4.423 & {$[0.358,0.565]$} & {$[3.858,4.065]$} \\
\hline 3320 & Agricultural & M & 60 & H-L-M & 2.4 & 4.938 & {$[3.166,4.801]$} & {$[0.137,1.772]$} \\
\hline 1321 & Municipal & $\mathrm{H}$ & 20 & H-L-H & 0.8 & 3.212 & {$[1.058,2.266]$} & {$[0.946,2.154]$} \\
\hline 2321 & Industrial & $\mathrm{H}$ & 20 & H-L-H & 0.8 & 4.423 & {$[0.087,4.299]$} & {$[0.124,4.336]$} \\
\hline 3321 & Agricultural & $\mathrm{H}$ & 20 & H-L-H & 0.8 & 4.938 & {$[2.032,4.546]$} & {$[0.392,2.906]$} \\
\hline 1322 & Municipal & $\mathrm{L}$ & 20 & H-M-L & 2.4 & 3.212 & {$[2.553,2.817]$} & {$[0.395,0.659]$} \\
\hline 2322 & Industrial & $\mathrm{L}$ & 20 & H-M-L & 2.4 & 4.423 & {$[4.047,4.256]$} & {$[0.167,0.376]$} \\
\hline 3322 & Agricultural & $\mathrm{L}$ & 20 & H-M-L & 2.4 & 4.938 & {$[0.542,4.078]$} & {$[0.860,4.396]$} \\
\hline 1323 & Municipal & M & 60 & H-M-M & 7.2 & 3.212 & {$[0.811,2.625]$} & {$[0.587,2.401]$} \\
\hline 2323 & Industrial & M & 60 & H-M-M & 7.2 & 4.423 & {$[2.370,2.910]$} & {$[1.513,2.053]$} \\
\hline 3323 & Agricultural & M & 60 & H-M-M & 7.2 & 4.938 & {$[2.874,4.022]$} & {$[0.916,2.064]$} \\
\hline 1324 & Municipal & $\mathrm{H}$ & 20 & H-M-H & 2.4 & 3.212 & {$[3.097,3.119]$} & {$[0.093,0.115]$} \\
\hline 2324 & Industrial & $\mathrm{H}$ & 20 & H-M-H & 2.4 & 4.423 & {$[2.321,3.201]$} & {$[1.222,2.102]$} \\
\hline 3324 & Agricultural & $\mathrm{H}$ & 20 & $\mathrm{H}-\mathrm{M}-\mathrm{H}$ & 2.4 & 4.938 & {$[3.064,3.905]$} & {$[1.033,1.874]$} \\
\hline 1325 & Municipal & $\mathrm{L}$ & 20 & $\mathrm{H}-\mathrm{H}-\mathrm{L}$ & 0.8 & 3.212 & {$[1.332,2.673]$} & {$[0.539,1.880]$} \\
\hline 2325 & Industrial & $\mathrm{L}$ & 20 & H-H-L & 0.8 & 4.423 & {$[0.597,4.261]$} & {$[0.162,3.826]$} \\
\hline 3325 & Agricultural & $\mathrm{L}$ & 20 & $\mathrm{H}-\mathrm{H}-\mathrm{L}$ & 0.8 & 4.938 & {$[1.108,4.456]$} & {$[0.482,3.830]$} \\
\hline 1326 & Municipal & M & 60 & H-H-M & 2.4 & 3.212 & {$[2.482,2.676]$} & {$[0.536,0.730]$} \\
\hline 2326 & Industrial & M & 60 & H-H-M & 2.4 & 4.423 & {$[0.553,1.948]$} & {$[2.475,3.870]$} \\
\hline 3326 & Agricultural & M & 60 & H-H-M & 2.4 & 4.938 & {$[1.731,2.663]$} & {$[2.275,3.207]$} \\
\hline 1327 & Municipal & $\mathrm{H}$ & 20 & $\mathrm{H}-\mathrm{H}-\mathrm{H}$ & 0.8 & 3.212 & {$[0.865,0.866]$} & {$[2.346,2.347]$} \\
\hline 2327 & Industrial & $\mathrm{H}$ & 20 & $\mathrm{H}-\mathrm{H}-\mathrm{H}$ & 0.8 & 4.423 & {$[0.790,4.205]$} & {$[0.218,3.633]$} \\
\hline 3327 & Agricultural & $\mathrm{H}$ & 20 & H-H-H & 0.8 & 4.938 & {$[3.320,3.577]$} & {$[1.361,1.618]$} \\
\hline
\end{tabular}

$y_{13 \mathrm{opt}}=0.212, y_{23 \mathrm{opt}}=0.211$, and $y_{33 \mathrm{opt}}=0.375 ; \alpha$-optimistic value to the net system benefit: $\bar{f}_{\mathrm{opt}}^{ \pm}=[154.335,235.704]$.

corresponding variables or data in the model. Then the model could be solved by the hybrid algorithm.

\section{Conclusions}

An inexact multistage stochastic chance constrained programming (IMSCCP) model is provided for water resources management, which integrates stochastic CCP proposed by Liu [21], multistage stochastic programming, and inexact stochastic programming. Compared with the existing IMSP with chance constraints, the IMSCCP model proposed in this study contains stochastic variables in the objective function or inexact data with multiply distributed stochastic boundaries. Then the IMSCCP model could be solved by using the hybrid algorithm. After solving the IMSCCP model, the maximum, which is the optimistic value to the net system benefit with a predetermined confidence level, could be obtained subject to the chance constraints with other confidence levels. 
Considering more real-world situations in the water resources management systems, such as water distribution from regulated reservoir(s), the expansion and development of the reservoir(s), and more uncertainties existing in many system components, further studies can resort to nonlinear programming combined with other uncertain variables such as fuzzy variable and $g_{\lambda}$ variable to solve the water resources management problems and handle more uncertainties. Moreover, research efforts might also be devoted to the wide application of IMSCCP model in the areas of ecological water requirement system management, waste management planning, electric-power system planning, and so on.

\section{Conflicts of Interest}

The authors declare that there are no conflicts of interest regarding the publication of this paper.

\section{Acknowledgments}

This work was supported by the National Natural Science Foundation of China (Grant no. 11626079), the Application Basic Research Plan Key Basic Research Project of Hebei Province (Grant no. 16964213D), the Natural Science Foundation of Hebei Province of China (Grant no. F2015402033), the Natural Science Foundation of the Hebei Education Department (Grants nos. QN2015116 and BJ2017031), the Innovation Fund for Postgraduates of Hebei Province in 2016 (Grant no. 222), and the Plan Project for Science and Technology in Handan City (Grants nos. 1528102058-5 and 1534201095-3).

\section{References}

[1] X. M. Liu, G. H. Huang, S. Wang, and Y. R. Fan, "Water resources management under uncertainty: factorial multi-stage stochastic program with chance constraints," Stochastic Environmental Research and Risk Assessment, vol. 30, no. 3, pp. 945957, 2016.

[2] J. Gao and F. You, "Optimal design and operations of supply chain networks for water management in shale gas production: MILFP model and algorithms for the water-energy nexus," AIChE Journal, vol. 61, no. 4, pp. 1184-1208, 2015.

[3] Z. Hu, Y. Chen, L. Yao, C. Wei, and C. Li, "Optimal allocation of regional water resources: From a perspective of equityefficiency tradeoff," Resources, Conservation and Recycling, vol. 109, pp. 102-113, 2016.

[4] T. Roach, Z. Kapelan, R. Ledbetter, and M. Ledbetter, "Comparison of robust optimization and info-gap methods for water resource management under deep uncertainty," Journal of Water Resources Planning and Management, vol. 142, no. 9, Article ID 04016028, 2016.

[5] M. G. Rojas-Torres, F. Nápoles-Rivera, J. M. Ponce-Ortega, M. Serna-González, G. Guillén-Gosálbez, and L. Jiménez-Esteller, "Multiobjective optimization for designing and operating more sustainable water management systems for a city in Mexico," AIChE Journal, vol. 61, no. 8, pp. 2428-2446, 2015.

[6] Y. Tu, X. Zhou, J. Gang, M. Liechty, J. Xu, and B. Lev, "Administrative and market-based allocation mechanism for regional water resources planning," Resources, Conservation and Recycling, vol. 95, pp. 156-173, 2015.

[7] J. Liu, Y. P. Li, G. H. Huang, and X. T. Zeng, "A dual-interval fixed-mix stochastic programming method for water resources management under uncertainty," Resources, Conservation and Recycling, vol. 88, pp. 50-66, 2014.

[8] Y. L. Xie, G. H. Huang, W. Li, J. B. Li, and Y. F. Li, "An inexact two-stage stochastic programming model for water resources management in Nansihu Lake Basin, China," Journal of Environmental Management, vol. 127, pp. 188-205, 2013.

[9] Y. P. Li, G. H. Huang, and S. L. Nie, "An interval-parameter multi-stage stochastic programming model for water resources management under uncertainty," Advances in Water Resources, vol. 29, no. 5, pp. 776-789, 2006.

[10] Y. Zhou, G. H. Huang, and B. Yang, "Water resources management under multi-parameter interactions: a factorial multistage stochastic programming approach," Omeg, vol. 41, no. 3, pp. 559-573, 2012.

[11] M. Q. Suo, Y. P. Li, G. H. Huang, Y. R. Fan, and Z. Li, "An inventory-theory-based inexact multistage stochastic programming model for water resources management," Mathematical Problems in Engineering, vol. 2013, Article ID 482095, 2013.

[12] F. Chen, G. H. Huang, and Y. R. Fan, "Inexact multistage fuzzystochastic programming model for water resources management," Journal of Water Resources Planning and Management, vol. 141, no. 11, Article ID 04015027, 2015.

[13] Y. P. Li, G. H. Huang, Z. F. Yang, and S. L. Nie, "IFMP: interval-fuzzy multistage programming for water resources management under uncertainty," Resources, Conservation and Recycling, vol. 52, no. 5, pp. 800-812, 2008.

[14] Y. P. Li, G. H. Huang, Y. F. Huang, and H. D. Zhou, "A multistage fuzzy-stochastic programming model for supporting sustainable water-resources allocation and management," Environmental Modelling and Software, vol. 24, no. 7, pp. 786-797, 2009.

[15] A. Charnes and W. W. Cooper, "Chance-constrained programming," Management Science, vol. 6, no. 1, pp. 73-79, 1959.

[16] R. Rossi, S. Armagan Tarim, B. Hnich, S. Prestwich, and C. Guran, "A note on Liu-Iwamura's dependent-chance programming," European Journal of Operational Research, vol. 198, no. 3, pp. 983-986, 2009.

[17] J. M. Grosso, C. Ocampo-Martínez, V. Puig, and B. Joseph, "Chance-constrained model predictive control for drinking water networks," Journal of Process Control, vol. 24, no. 5, pp. 504-516, 2014.

[18] H. Wu, M. Shahidehpour, Z. Li, and W. Tian, "Chanceconstrained day-ahead scheduling in stochastic power system operation," IEEE Transactions on Power Systems, vol. 29, no. 4, pp. 1583-1591, 2014.

[19] Y. C. Han, G. H. Huang, and C. H. Li, "An interval-parameter multi-stage stochastic chance-constrained mixed integer programming model for inter-basin water resources management systems under uncertainty," in Proceedings of the 5th International Conference on Fuzzy Systems and Knowledge Discovery, FSKD '08, pp. 146-153, IEEE, Shandong, China, October 2008.

[20] B. Liu and K. Iwamura, "Fuzzy programming with fuzzy decisions and fuzzy simulation-based genetic algorithm," Fuzzy Sets and Systems, vol. 122, no. 2, pp. 253-262, 2001.

[21] B. D. Liu, Uncertain Programming, Wiley, New York, NY, USA, 1999. 
[22] H. Ke and B. Liu, "Fuzzy project scheduling problem and its hybrid intelligent algorithm," Applied Mathematical Modelling. Simulation and Computation for Engineering and Environmental Systems, vol. 34, no. 2, pp. 301-308, 2010.

[23] J. W. Gao, J. H. Zhao, and X. Y. Ji, "Fuzzy chance-constrained programming for capital budgeting problem with fuzzy decisions," in Proceedings of the 2nd International Conference Fuzzy Systems and Knowledge Discovery, FSKD '05, vol. 3613, pp. 304311, IEEE, Changsha, China, August 2005.

[24] J. Zhou and B. Liu, "New stochastic models for capacitated location-allocation problem," Computers and Industrial Engineering, vol. 45, no. 1, pp. 111-125, 2003.

[25] R. Zhao and B. Liu, "Stochastic programming models for general redundancy-optimization problems," IEEE Transactions on Reliability, vol. 52, no. 2, pp. 181-191, 2003.

[26] H. Zhang, M. Ha, and H. Xing, "Chance-constrained programming on sugeno measure space," Expert Systems with Applications, vol. 38, no. 9, pp. 11527-11533, 2011.

[27] M. Ha, Z. Gao, and X. Wang, "Managing water supply risk through an option contract in uncertain environment," Journal of Uncertain Systems, vol. 10, no. 2, pp. 114-123, 2016.

[28] Y. P. Li, G. H. Huang, S. L. Nie, and L. Liu, "Inexact multistage stochastic integer programming for water resources management under uncertainty," Journal of Environmental Management, vol. 88, no. 1, pp. 93-107, 2008.

[29] D. P. Loucks, J. R. Stedinger, and D. A. Haith, Water resource systems planning and analysis, Prentice-Hall, Upper Saddle River, NJ, USA, 1981.

[30] G. H. Huang and D. P. Loucks, "An inexact two-stage stochastic programming model for water resources management under uncertainty," Civil Engineering and Environmental Systems, vol. 17, no. 2, pp. 95-118, 2000.

[31] A. Charnes, W. W. Cooper, and M. J. L. Kirby, "Chanceconstrained programming: an extension of statistical method," Optimizing Methods in Statistics, pp. 391-402, 1971. 

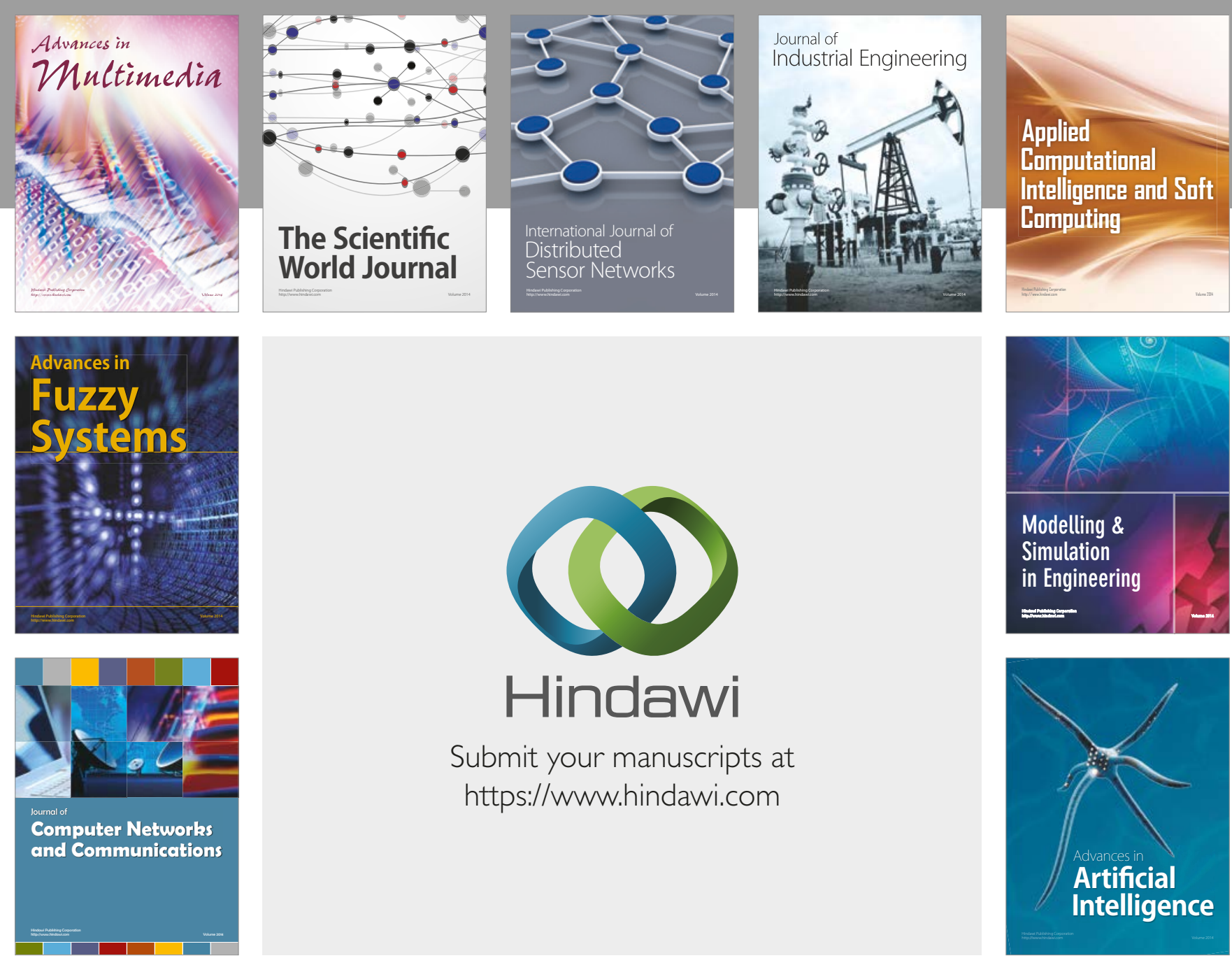

\section{Hindawi}

Submit your manuscripts at

https://www.hindawi.com
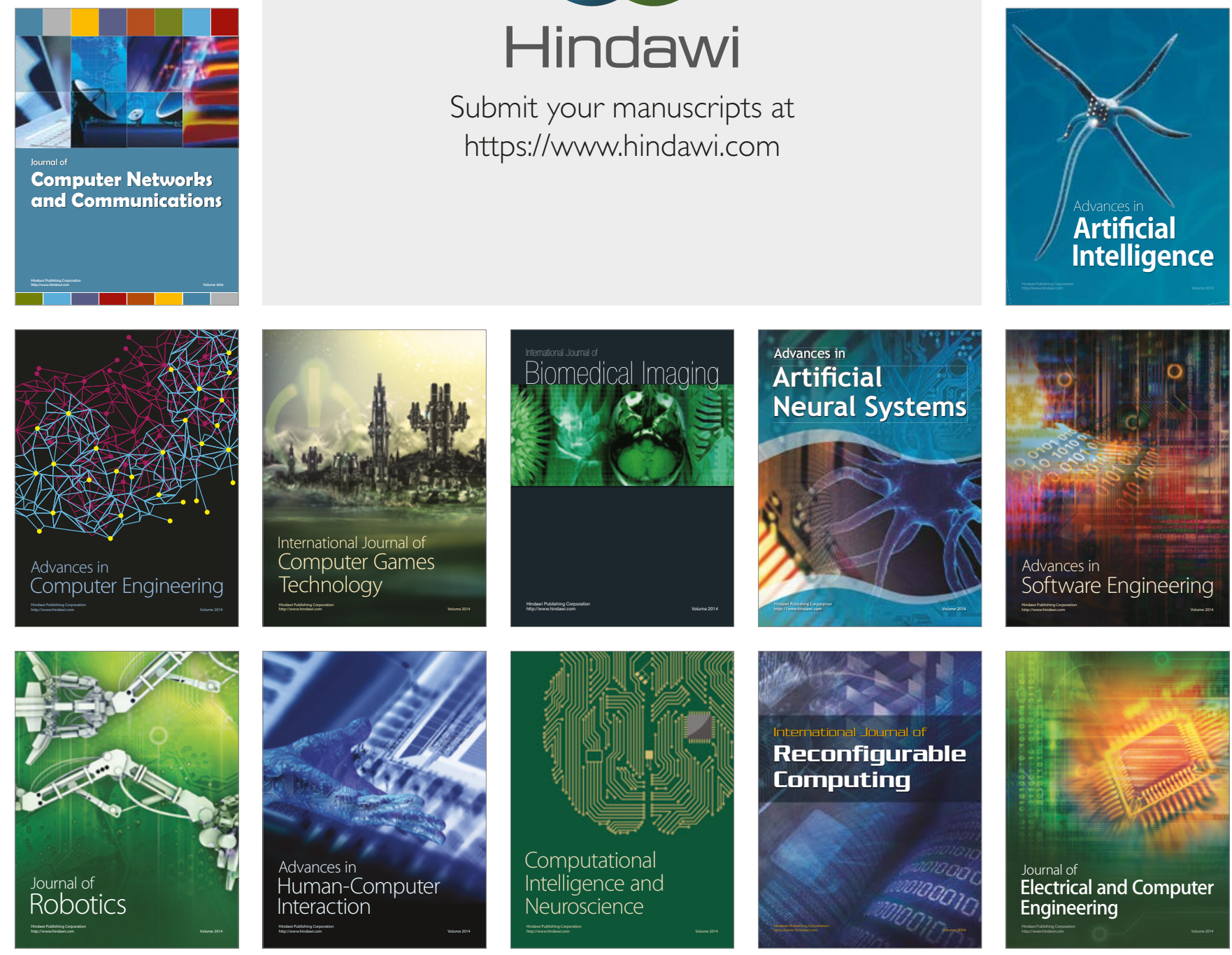